\title{
In-medium Yang-Mills equations: a derivation and canonical quantization
}

\author{
M. K. Djongolov ${ }^{1)}$, S.Pisov ${ }^{2)}$, V.Rizov ${ }^{2)}$ \\ 1) Department of Physics and Astronomy, University of \\ Tennessee, \\ e-mail: martin@spock.phys.utk.edu \\ 2) University of Sofia, Faculty of Physics, \\ e-mail: pisov@phys.uni-sofia.bg; rizov@phys.uni-sofia.bg
}




\begin{abstract}
The equations for a Yang-Mills field in a medium are derived in the approximation of linear response to an external field. Introducing tensors of generalized susceptibilities, the in-medium equations are written in a form similar to the in-medium Maxwell equations. The non-Abelian character of the gauge group $G$ is reflected in the presence of susceptibility tensors with no counterpart in electrodynamics and the necessity to define modified "electric" and "magnetic" fields (apart from the "color" induction vectors). which enter the in-medium equations. The latter reduce to $\operatorname{dim} G$ copies of Maxwell in-medium equations in the approximation up to second order in the gauge coupling constant. For a medium uniformly moving, a canonical quantization is performed for a family of Fermi-like gauges in the case of constant and diagonal (in the group indices) tensors of "electric" permittivity and "magnetic" permeability. The physical subspace is defined and the gauge field propagator is evaluated for a particular choice of the gauge. It is applied firstly for evaluation of the cross-section of elastic quark scattering in the Born approximation. Possible applications to Cherenkov-type gluon radiation are commented briefly.
\end{abstract}

\title{
1. Introduction
}

In some developments of the Yang-Mills theories one arrives at situations when the encountered states can be effectively described as media, possessing non-Abelian polarization properties. We list a few of these examples for sake of brevity.

Among the first prototypes for a non-Abelian polarizable medium one may take the QCD vacuum state in the aspect considered by Savvidy [1], Fukuda and Kazama [2], Adler [3], Nielsen and Olesen [4]. In [5] and [6] Baker and Zachariasen used the YangMills vacuum as a polarizable medium in order to derive a confining potential. They also solved the Dyson equations for the gluon propagator $\Delta_{\mu \nu}(q)$ for $q^{2} \rightarrow 0$ evaluating the color susceptibility of the vacuum in the weak field approximation. The polarization properties of the non-Abelian $S U(2)$ vacuum were exploited in [7, 8] for an approximate classical description of some non-perturbative effects such as solutions corresponding to non-vanishing gauge field "condensates".

Another and more recent set of examples comes from the theoretical study of some not yet confirmed states of the hadron matter - hadron matter at very high densities and quark-gluon plasma (QGP) at very high temperatures where one expects the quarks and gluons to be in a deconfined phase. In a systematic study performed in the framework of kinetic theory the color conductivity and other transport coefficients of non-Abelian plasma were derived by Heinz in $[9,10]$ in the linear response approximation. The response of QGP beyond the linear approximation was considered in [11] yielding a color dielectric susceptibility modified by the non-linear corrections. Typical non-Abelian medium effects on the color dielectric constant $\epsilon$ were further investigated in [12] also on the base of kinetic theory.

The approach of Heinz has been generalized in $[13,14]$ to include the effects of fluctuations in deriving effective transport equations for QCD. A clear review of the methods of semi-classical transport theory for non-Abelian plasma can be found in [15]. 
From the analysis of the phenomenon of color superconductivity of hadron matter at high density [16, 17], it has been found that the medium surrounding the liberated quarks exhibits a polarization behavior characterized by an effective color dielectric constant $\epsilon \gg 1$, the magnetic permeability remaining close to one. The estimate $\epsilon \gg 1$ has also been obtained in [18] evaluating the photon self-energy for a color superconductor. The case of two-flavor color superconductor with prescribed dielectric susceptibility and magnetic permeability of the medium was considered by D. Litim and C. Manuel [19] applying the methods of transport theory and extending the results of Rischke, Son and Stephanov to finite temperature.

Finally, we mention models such as the chromodielectric model proposed by Friedberg and Lee [20, 21] and developed in [22, 23, 24, 25, 26, 27]. Aimed to describe an effective mechanism for the confinement phenomena, this model exploits a dielectric function depending on a classical field in order to account for the interaction of the QCD matter. Also in $[28,29]$ Dremin introduced a refraction coefficient $n$ phenomenologically for a hadron medium, exploiting the relation between $n$ and the elastic forward scattering amplitude of light derived in classical electrodynamics. Among other applications, for a quark-parton medium he used the appearance of a so postulated $n$ to consider the emission of Cherenkov-type gluons (for $n>1$ ) in order to explain the ring-like structures observed in hadron-hadron or central nucleus-nucleus collisions as generated by gluon jets produced in such processes (see e.g.[30]).

Having in mind these examples, our view-point is that, if in the interactions of a medium with Yang-Mills fields one encounters manifestations of its polarization-type response to the gauge fields, then it is worth developing an in-medium Yang-Mills theory and apply it to situations where such a response is found to be essential (like, e.g. the quark-gluon plasma).

In this paper we derive the classical Yang-Mills equations for a gauge group $G$ in a medium in the case when the medium responses linearly to an external gauge field (i.e. we derive the analogs of the macroscopic Maxwell equations for a gauge theory) and perform a canonical quantization of the arising theory. In the derivation of the classical in-medium gauge field equations we follow the method of Akhiezer and Peletminsky [31] in their derivation of the macroscopic Maxwell equations. In Section 2 we introduce some of the notations and write down the equations for a system of interacting spinor and gauge fields in the presence of a weak external gauge field. In Section 3 these equations are averaged by means of a non-equilibrium statistical operator in the linear approximation with respect to the external field, and the averaged "color" electric and magnetic fields $\left(E^{a}, B^{a}\right)$ are introduced. There appear three types of (generalized) conductivity tensors defined in terms of the relevant retarded Green's functions. The first is a straightforward generalization to the Yang-Mills case of the "classical electrodynamic" conductivity tensor, while the second and the third one appear due to the (eventually) non-commutative character of the gauge group. In Section 4 the relevant polarization tensors, characterizing the medium, are defined, two of them having direct analogs in the classical case, and other two originating from the non-commutativity 
of the gauge group. Assuming a linear relation between the polarization tensors and the fields $\left(E^{a}, B^{a}\right)$, we define the (non-Abelian) susceptibility tensors. In Section 5 we define the generalized electric and magnetic inductions, the (generalized) magnetic permeability and electric permittivity tensors $\mu^{a b}, \epsilon^{a b}$, and write down the derived inmedium Yang-Mills equations. As in the standard case, four of these equations describe the "color" induction vectors. In order to write the remaining equations, we define modified "color" fields $\left(E^{\prime a}, B^{\prime a}\right)$ obtained from $\left(E^{a}, B^{a}\right)$ by means of the "non-Abelian" susceptibility tensors. In Section 6 we perform an approximation up to second order in the gauge coupling constant which simplifies the in-medium equations significantly reducing them to a form resembling closely the Maxwell in-medium equations. Defining the constitutive equations for the medium, we obtain relations between $\epsilon^{a b}, \mu^{a b}$, and the conductivity tensors in the approximation considered. These relations appear as natural generalizations of their analogs in the classical macroscopic electrodynamics. We follow Brevik and Lautrup [32] and Nakanishi [33, 34] in their treatment of the electromagnetic field which avoids work with constrained systems. Although in [32] one deals with in-medium electromagnetic field, and [33, 34, 35] consider the electromagnetic field in vacuum, these papers use a common approach, namely a Lagrangian formulation involving an auxiliary scalar field whose action on the Fock space is designed to separate the physical subspace. For a medium moving uniformly, Section 5 provides a Lagrangian formulation of the previously obtained equations introducing a set of $\operatorname{dim}(G)$ auxiliary fields $B^{a}$. For sake of simplicity we consider constant and diagonal tensors $\epsilon$ and $\mu$. From the equal time commutation relations, in Section 6 we evaluate the commutators at arbitrary space-time points. In Section 7 the fields' Fourier transforms are introduced. In the resulting Fock space a physical subspace is defined by means of the operators $B^{a}$. Finally, for a particular choice of the gauge we evaluate the gauge field propagator. As a first application the cross-section for elastic quark-quark scattering is given in the Born approximation. It is noticed that the in-medium Yang-Mills equations considered here provide a natural framework for the Dremin's approach to explain the specific ringlike structures in hadron processes as resulting from a Cherenkov-type gluon radiation. Other applications, like, e.g. color superconductivity, lie beyond the scope of this paper.

\section{Field equations}

Let $G$ be a compact $r$-dimensional simple matrix Lie group. Let $T^{a}(a=1, \ldots, r)$ be a basis of hermitian matrices in its Lie algebra $\mathcal{G}$ with commutation relations

$$
\left[T^{a}, T^{b}\right]=i f^{a b c} T^{c}
$$

and normalized by

$$
\operatorname{tr}\left(T^{a} T^{b}\right)=\frac{1}{2} \delta^{a b}
$$

Lower case Latin letters $a, b, c, \ldots$ are used for the group /algebra indices and here and below summation over repeated indices is understood. 
Let $\hat{\Psi}(x)$ be a quantized spinor field with values in a vector space $\mathcal{W}$, i.e. $\hat{\Psi}(x) \in \hat{\mathcal{S}} \otimes \mathcal{W}$, where $\hat{\mathcal{S}}$ is the space of operator-valued spinors over Minkowski space $M, x \in M$. We suppose that $\mathcal{W}$ carries a linear representation $\omega \rightarrow R(\omega)$ of $\mathrm{G}, \omega \in G$, and that $\mathcal{W}$ is equipped with a scalar product such that $R(\omega)$ is unitary.

Given a quantized Yang-Mills gauge potential $\hat{\mathcal{A}}_{\mu}(x)=\hat{A}_{\mu}^{a}(x) T^{a}(\mu=0, \ldots, 3)$, the gauge covariant derivative of the spinor field $\hat{\Psi}(x)$ reads (in coordinates $\left(x^{\mu}\right)=\left(x^{0}, \vec{x}\right)$ )

$$
\mathcal{D}_{\mu}(\hat{\mathcal{A}}) \hat{\Psi}(x)=\partial_{\mu} \hat{\Psi}(x)+i g R\left(\hat{\mathcal{A}}_{\mu}(x)\right) \hat{\Psi}(x)=\partial_{\mu} \hat{\Psi}(x)+i g \hat{A}_{\mu}^{a}(x) R\left(T^{a}\right) \hat{\Psi}(x),
$$

where $\mathcal{D}_{\mu}(\hat{\mathcal{A}})$ denotes covariant differentiation with respect to $\hat{\mathcal{A}}_{\mu}$, and $g$ is a gauge coupling constant $\left(R\left(T^{a}\right)\right.$ stands for the generators of $\mathrm{G}$ in the representation $R$ ). We suppose, in addition, that the spinor field $\hat{\Psi}$ is put in an external (classical) gauge field $f_{\mu \nu}$ with gauge potential $a_{\mu}=a_{\mu}^{c} T^{c}$, i.e.

$$
f_{\mu \nu}=\partial_{\mu} a_{\nu}-\partial_{\nu} a_{\mu}+i g\left[a_{\mu}, a_{\nu}\right],
$$

and $f_{\mu \nu}$ is determined by an external current

$$
j^{\mu}=j^{a, \mu} T^{a},
$$

so that

$$
\mathcal{D}_{\mu}(a) f^{\mu \nu}=\partial_{\mu} f^{\mu \nu}+i g\left[a_{\mu}, f^{\mu \nu}\right]=g j^{\nu} .
$$

Fixing the units such that $\hbar=c=1$, the Minkowski metric $\left(g_{\mu \nu}\right)=$ $\operatorname{diag}(1,-1,-1,-1)$, and denoting the operators $\left(\partial_{n}\right)=\left(-\partial^{n}\right)=\vec{\nabla}$, we also introduce the components of the analogs of the electric and magnetic fields

$$
\begin{aligned}
\hat{E}^{a, k} & =-\hat{\mathcal{F}}^{a, 0 k}, \\
\hat{B}^{a, k} & =-\frac{1}{2} \epsilon^{k m j} \hat{\mathcal{F}}^{a, m j},
\end{aligned}
$$

where $a=1, \ldots, r ; k=1,2,3, \epsilon^{123}=1$ and

$$
\hat{\mathcal{F}}^{\mu \nu}=\partial^{\mu} \hat{\mathcal{A}}^{\nu}-\partial^{\nu} \hat{\mathcal{A}}^{\mu}+i g\left[\hat{\mathcal{A}}^{\mu}, \hat{\mathcal{A}}^{\nu}\right]=\hat{F}^{a, \mu \nu} T^{a} .
$$

The gauge potentials $\hat{\mathcal{A}}_{\mu}$ are taken in the temporal gauge, $\hat{\mathcal{A}}_{0}=0$, satisfying $\partial_{k} \hat{\mathcal{A}}_{k}=0$. We fix also $\partial_{\mu} a^{\mu}=0$. The equations for $\hat{\mathcal{A}}(x)$ are

$$
\begin{aligned}
& \partial_{0} \hat{E}^{a, k}(x)=-\epsilon^{k m j}\left(\mathcal{D}^{m}(\hat{\mathcal{A}}) \hat{B}^{j}(x)\right)^{a}-g\left(\hat{I}^{a, k}(x)+j^{a, k}\left(x^{0}, \vec{x}\right)\right), \\
& \partial_{0} \hat{B}^{a, k}(x)=\epsilon^{k m j}\left(\mathcal{D}^{m}(\hat{\mathcal{A}}) \hat{E}^{j}(x)\right)^{a}, \\
& \left(\mathcal{D}^{k}(\hat{\mathcal{A}}) \hat{B}^{k}(x)\right)^{a}=0, \\
& \left(\mathcal{D}^{k}(\hat{\mathcal{A}}) \hat{E}^{k}(x)\right)^{a}=g\left(\hat{I}^{a, 0}(x)+j^{a, 0}\left(x^{0}, \vec{x}\right)\right) .
\end{aligned}
$$

Here $\hat{I}_{\nu}(x)=\hat{I}_{\nu}^{a}(x) T^{a}$ is the spinor current with components

$$
\hat{I}_{\nu}^{a}(x)=\hat{\Psi}^{\dagger}(x) \gamma_{\nu} R\left(T^{a}\right) \hat{\Psi}(x) .
$$

The equation for $\hat{\Psi}(x)$ reads

$i \gamma^{0} \partial_{0} \hat{\Psi}(x)+i \gamma^{n} \mathcal{D}_{n}(\hat{\mathcal{A}}) \hat{\Psi}(x)+i \gamma^{0} \mathcal{D}_{0}(a) \hat{\Psi}(x)+i \gamma^{k} \mathcal{D}_{k}(a) \hat{\Psi}(x)-m \hat{\Psi}(x)=0$, 
where $\gamma^{\lambda} \gamma^{\mu}+\gamma^{\mu} \gamma^{\lambda}=2 g^{\lambda \mu} \mathbf{1}$. It follows from equation (8) that

$$
\mathcal{D}^{\mu}(\hat{\mathcal{A}}) \hat{I}_{\mu}=-i g\left[a^{\mu}, \hat{I}_{\mu}\right] \text {. }
$$

In absence of the exterior gauge field the energy functional has the form

$$
\hat{H}_{0}=\int d x^{3}\left\{\frac{1}{2} \sum_{a=1}^{r}\left(\vec{E}^{a} \cdot \vec{E}^{a}+\vec{B}^{a} \cdot \vec{B}^{a}\right)+\hat{\Psi}^{\dagger} \gamma^{0}\left(-i \gamma^{k} \mathcal{D}_{k}(\hat{\mathcal{A}}) \hat{\Psi}+m \hat{\Psi}\right)\right\}
$$

\section{Response of the system to the exterior gauge field}

\subsection{Density operator and averaged field equations}

We suppose that at infinite past $\left(x^{0}=-\infty\right)$ in the absence of the exterior field the system of interacting fermion and gauge fields is in equilibrium at temperature $1 / \beta$, described by the density operator $\hat{\rho}_{0}=\exp (-\beta \hat{H})$. At time $x^{0}=-\infty$ the exterior field $a^{\mu}$ is switched in adiabatically and the system is described by an non-equilibrium density operator $\hat{\rho}\left(x^{0}\right)$ satisfying

$$
i \partial_{0} \hat{\rho}\left(x^{0}\right)=\left[\hat{H}\left(x^{0}\right), \hat{\rho}\left(x^{0}\right)\right],
$$

with the initial condition $\hat{\rho}(-\infty)=\hat{\rho}_{0}$. The total Hamiltonian is

$$
\hat{H}\left(x^{0}\right)=\hat{H}_{0}+g \hat{V}\left(x^{0}\right),
$$

where $\hat{V}\left(x^{0}\right)$ describes the interaction of the fermions with the gauge potential $a_{\mu}^{c}$,

$$
\hat{V}\left(x^{0}\right)=\int d^{3} x \hat{I}^{c, \mu}(\vec{x}) a_{\mu}^{c}\left(x^{0}, \vec{x}\right) .
$$

It is convenient to go to the interaction (Dirac) representation, defined by the splitting of the Hamiltonian

$$
\hat{H}=\hat{H}\left(x^{0}\right)=\hat{H}_{0}+g \hat{V}\left(x^{0}\right) .
$$

In this representation the density operator $\hat{\rho}_{\mathcal{D}}\left(x^{0}\right)$ satisfies

$$
i \partial \hat{\rho}_{\mathcal{D}}\left(x^{0}\right)=g\left[\hat{V}_{\mathcal{D}}\left(x^{0}\right), \hat{\rho}_{\mathcal{D}}\left(x^{0}\right)\right],
$$

and the initial condition $\hat{\rho}_{\mathcal{D}}(-\infty)=\hat{\rho}_{0}$, where

$$
\hat{V}_{\mathcal{D}}\left(x^{0}\right)=\int d^{3} x \hat{J}^{\mu}(x) a_{\mu}(x),
$$

and $\hat{J}^{\mu}$ is the fermion current in the Dirac representation

$$
\hat{J}^{\mu}(x) \equiv \hat{I}_{\mathcal{D}}^{\mu}(x)=\exp \left(i x^{0} \hat{H}_{0}\right) \hat{I}^{\mu}(x) \exp \left(-i x^{0} \hat{H}_{0}\right) .
$$

Combining equation (12) and the initial condition in the integral equation

$$
\hat{\rho}_{\mathcal{D}}\left(x^{0}\right)=\hat{\rho}_{0}-i g \int_{-\infty}^{x^{0}} d t\left[\hat{V}_{\mathcal{D}}(t), \rho_{\mathcal{D}}(t)\right]
$$

after the first iteration one finds the well known representation

$$
\hat{\rho}_{\mathcal{D}}\left(x^{0}\right)=\hat{\rho}_{0}-i g \int_{-\infty}^{+\infty} d t \theta\left(x^{0}-t\right)\left[\hat{V}_{\mathcal{D}}(t), \hat{\rho}_{0}\right] .
$$


In other terms, the linear approximation of $\hat{\rho}_{\mathcal{D}}$ with respect to the exterior field is

$$
\hat{\rho}_{\mathcal{D}}\left(x^{0}\right)=\hat{\rho}_{0}-i g \int d^{4} y \theta\left(x^{0}-y^{0}\right)\left[\hat{J}_{\mu}(y), \hat{\rho}_{0}\right] a^{\mu}(y) .
$$

Averaging equations (3-6) by means of $\hat{\rho}_{\mathcal{D}}$ one find that the mean values

$$
A_{\mu}(x)=<\hat{A}_{\mu}(x)>\equiv A_{\mu}^{a}(x) T^{a}
$$

and

$$
F_{\mu \nu}(x)=\partial_{\mu} A_{\nu}(x)-\partial_{\nu} A_{\mu}(x)+i g\left[A_{\mu}(x), A_{\nu}(x)\right] \equiv F_{\mu \nu}^{a}(x) T^{a}
$$

obey the equations

$$
\begin{aligned}
& \mathcal{D}^{\mu}(A) F_{\mu \nu}=g J_{\nu}-g S_{\nu}, \\
& \mathcal{D}^{\mu}(A) * F_{\mu \nu}=g R_{\nu},
\end{aligned}
$$

where

$$
\begin{aligned}
J_{\nu}= & <\hat{J}_{\nu}>\equiv J_{\nu}^{a} T^{a}, \\
S_{\nu}= & i\left\{\partial^{\mu}\left(<\left[\hat{A}_{\mu}, \hat{A}_{\nu}\right]>-\left[A_{\mu}, A_{\nu}\right]\right)+\right. \\
& \left.+<\left[\hat{A}^{\mu}, \hat{F}_{\mu \nu}\right]>-\left[A^{\mu}, F_{\mu \nu}\right]\right\} \equiv S_{\nu}^{a} T^{a}, \\
& =\frac{1}{R_{\nu}} \epsilon_{\nu \mu \sigma \tau} i\left\{\partial^{\mu}\left(<\left[\hat{A}^{\sigma}, \hat{A}^{\tau}\right]>-\left[A^{\sigma}, A^{\tau}\right]\right)+\right. \\
& \left.+<\left[\hat{A}^{\mu}, \hat{F}^{\sigma \tau}\right]>-\left[A^{\mu}, F^{\sigma \tau}\right]\right\} \equiv R_{\nu}^{a} T^{a}
\end{aligned}
$$

(the same notation for operator commutator and Lie algebra commutator should not be confusing). The dual of a tensor $F_{\mu \nu}$ is $* F_{\mu \nu} \equiv \frac{1}{2} \epsilon_{\mu \nu \sigma \tau} F^{\sigma \tau}\left(\epsilon_{0123}=-\epsilon^{0123}=-1\right)$ and $\mathcal{D}^{\mu}(A) F_{\mu \nu}=\partial^{\mu} F_{\mu \nu}+i g\left[A^{\mu}, F_{\mu \nu}\right]$.

\subsection{Retarded Green's functions and tensors of conductivity}

For the mean value of an operator $A$ one obtains in the first approximation

$$
\begin{aligned}
& <\hat{A}\left(x^{0}, \vec{x}\right)>\equiv \operatorname{tr}\left(\hat{A}\left(x^{0}, \vec{x}\right) \rho_{\mathcal{D}}\left(x^{0}\right)\right)= \\
& =<\hat{A}\left(x^{0}, \vec{x}\right)>_{0}-i g \int_{-\infty}^{+\infty} d t<\left[\hat{A}\left(x^{0}, \vec{x}\right), \hat{V}_{\mathcal{D}}\left(x^{0}\right)\right]>_{0} \theta\left(x^{0}-t\right)
\end{aligned}
$$

Using the form of $\hat{V}_{\mathcal{D}}$ from equation (13) and the notation

$$
G_{A, J_{\mu}}(x, y)=-i \theta\left(x^{0}-y^{0}\right)<\left[\hat{A}(x), \hat{J}_{\mu}(y)\right]>_{0}
$$

for the retarded Green's function, one rewrites equation (20) as

$$
<\hat{A}(x)>=<\hat{A}(x)>_{0}+g \int d^{4} y G_{A, J_{\mu}}(x, y) a^{\mu}(y) .
$$

For two operators $\hat{A}(x)$ and $\hat{B}(x)$ which are covariant under space translations the corresponding Green's function $G_{A B}(x, y)=-i \theta(x-y)<[\hat{A}(x), \hat{B}(y)]>_{0}$ depends only on $x-y$ and in this case we shall simply write $G_{A B}(x, y) \equiv G_{A B}(x-y)$. Let $\hat{A}$ and $\hat{B}$ transform under the time reflection antiunitary operator $U$ according to

$$
U \hat{A}(x) U^{-1}=\epsilon_{A} \hat{A}^{*}\left(i_{t} x\right), U \hat{B}(x) U^{-1}=\epsilon_{B} \hat{B}^{*}\left(i_{t} x\right)
$$


where $^{*}$ denotes hermitian conjugation, $i_{t} x=\left(-x^{0}, \vec{x}\right)$, and $\left|\epsilon_{A}\right|=\left|\epsilon_{B}\right|=1$. If, in addition, $\hat{A}$ and $\hat{B}$ are hermitian, the invariance under time reflection and space translations implies the property

$$
G_{A B}\left(k_{0}, \vec{k}\right)=\epsilon_{A} \epsilon_{B} G_{A B}\left(k_{0},-\vec{k}\right)
$$

of the Fourier transform of $G_{A B}$

$$
G_{A B}=\int e^{i k_{o} x_{0}-i \vec{k} \cdot \vec{x}} G_{A B}\left(x_{0}, \vec{x}\right) d x_{o} d^{3} x
$$

(denoted by the same symbol). Substituting in equation (22) the components $\hat{J}_{\mu}^{a}(x)$ of the fermionic current from (14), (7) we obtain

$$
J_{\mu}^{a}(x)=<\hat{J}_{\mu}^{a}(x)>=g \int d^{4} y G_{\mu \nu}^{a b}(x-y) a^{b, \nu}(y)
$$

$a=1, \ldots, r$, where

$$
G_{\mu \nu}^{a b}(x-y)=-i \theta\left(x^{0}-y^{0}\right)<\left[\hat{J}_{\mu}^{a}(x), \hat{J}_{\nu}^{b}(x)\right]>_{0}
$$

and we have used the fact that for a neutral medium in equilibrium the mean values $<J_{\mu}^{\hat{a}(x)}>_{0}=0$.

The mean values which enter $S_{\nu}$ and $R_{\nu}$ may be expressed by the corresponding Green's functions, namely

$$
\begin{aligned}
& \left.i \partial^{\mu}<\left[\hat{A}_{\mu}, \hat{A}_{\nu}\right]^{a}>+i<\hat{A}^{\mu}, \hat{F}_{\mu \nu}\right]^{a}>=g \int d^{4} y K_{\nu \lambda}^{a b}(x-y) a^{b, \lambda}(y), \\
& \left.\frac{1}{2} \epsilon_{\nu \mu \sigma \tau} i\left\{\partial^{\mu}<\left[\hat{A}^{\sigma}, \hat{A}^{\tau}\right]^{a}>+<\hat{A}^{\mu}, \hat{F}_{\sigma \tau}\right]^{a}>\right\}= \\
& =g \int d^{4} y L_{\nu \lambda}^{a b}(x-y) a^{b, \lambda}(y)
\end{aligned}
$$

with

$$
\begin{aligned}
& K_{\nu \lambda}^{a b}(x-y)=-i \theta\left(x_{0}-y_{0}\right) i\left\{\partial^{\mu}<\left[\left[\hat{A}_{\mu}(x), \hat{A}_{\nu}(x)\right]^{a}, \hat{J}_{\lambda}^{b}(y)\right]>_{0}+\right. \\
& \left.+<i\left[\left[\hat{A}^{\mu}(x), \hat{F}_{\mu \nu}(x)\right]^{a}, \hat{J}_{\lambda}^{b}(y)\right]>_{0}\right\} \\
& L_{\nu \lambda}^{a b}(x-y)=-i \theta\left(x_{0}-y_{0}\right) \frac{i}{2} \epsilon_{\nu \mu \sigma \tau}\left\{\partial^{\mu}<\left[\left[\hat{A}^{\sigma}(x), \hat{A}^{\tau}(x)\right]^{a}, \hat{J}_{\lambda}^{b}(y)\right]>_{0}+\right. \\
& \left.+<\left[\left[\hat{A}^{\mu}(x), \hat{F}^{\sigma \tau}(x)\right]^{a}, \hat{J}_{\lambda}^{b}(y)\right]>_{0}\right\} .
\end{aligned}
$$

The mean values (24) and (25) evaluated by means of $\hat{\rho}_{0}$ vanish because Lorentz covariance and invariance under space translations imply that there is no non-zero 4(pseudo) vector with values in the Lie algebra $\mathcal{G}$. The components $G_{\mu \nu}^{a b}$ in equation (23) will be said to form the exterior conductivity tensor, by analogy with classical electrodynamics. Since the current $S_{\nu}$ and $R_{\nu}$ are due to the non-Abelian character of the gauge group $G$, the components $K_{\nu \lambda}^{a b}$ and $L_{\nu \lambda}^{a b}$ will be said to form the non-Abelian conductivity tensor and pseudotensor, respectively. 


\section{Tensors of generalized susceptibilities}

Proceeding in analogy with classical electrodynamics [31] we introduce the analog of polarization tensor

$$
\mathcal{P}_{\mu \nu}(x)=-\mathcal{P}_{\nu \mu}(x)=\mathcal{P}_{\mu \nu}^{a} T^{a},
$$

through a gauge covariant relation between $\mathcal{P}_{\mu \nu}$ and the averaged current $J_{\nu}$,

$$
J_{\nu}(x)=\mathcal{D}^{\mu} \mathcal{P}_{\mu \nu}(x)=\partial^{\mu} \mathcal{P}_{\mu \nu}(x)-i g\left[A^{\mu}(x), \mathcal{P}_{\mu \nu}(x)\right],
$$

the covariant differentiation being with respect to $A_{\mu}=<\hat{A}_{\mu}>$. Equation (26) is the substitute of $J_{\nu}=\partial^{\mu} P_{\mu \nu}$ in the case of electrodynamics. We define also the corresponding generalizations of the electric and magnetic polarizations, respectively, as $\mathcal{G}$-valued 3 -vectors $\vec{P}$ and $\vec{M}$,

$$
\begin{aligned}
P^{a, n} & =\mathcal{P}^{a, 0 n}, \\
M^{a, n} & =-\frac{1}{2} \epsilon^{n l j} \mathcal{P}^{a, l j} .
\end{aligned}
$$

$(a=1, \ldots, r)$. Written in components, the defining equation (26) reads

$$
\begin{aligned}
& J^{a, n}= \partial_{0} P^{a, n}+\epsilon^{n m j}\left(\partial_{m} M^{a, j}+g f^{a b c} A^{b, m} M^{c, j}\right) \equiv \\
& \equiv \partial_{0} P^{a, n}+(\overrightarrow{\mathcal{D}}(A) \times \vec{M})^{a, n}, \\
& J^{a, 0}=-\vec{\nabla} \cdot \vec{P}^{a}-g f^{a b c}\left(A^{b, n} P^{c, n}\right) \equiv-(\overrightarrow{\mathcal{D}}(A) \cdot \vec{P})^{a}, \\
& \overrightarrow{\mathcal{D}}(A) \cdot \vec{P}^{a}=\partial_{n} P^{a, n}+f^{a b c} A^{b, n} P^{c, n}, \\
&\overrightarrow{\mathcal{D}}(A) \times \vec{M})^{a, n}=\epsilon^{n k l}\left[\partial_{k} M^{a, l}+f^{a b c} A^{b, k} M^{c, l}\right]
\end{aligned}
$$

$\left(\vec{P}^{a}=\left(P^{a, n}\right), \vec{M}^{a}=\left(M^{a, n}\right)\right)$. Applying again the analogy with the case of electrodynamics, we fix the arbitrariness in $\mathcal{P}_{\mu \nu}$ assuming a linear relation between (the Fourier transformations of) $\vec{P}^{a}$ and $\vec{M}^{a}$, on the one hand, and the averaged "electric" and "magnetic" fields, $\vec{E}^{a}$ and $\vec{B}^{a}$, on the other:

$$
\begin{aligned}
\vec{P}^{a}\left(k_{0}, \vec{k}\right) & =\kappa^{a b}\left(k_{0}, \vec{k}\right) \vec{E}^{b}\left(k_{0}, \vec{k}\right), \\
\vec{M}^{a}\left(k_{0}, \vec{k}\right) & =\psi^{a b}\left(\left(k_{0}, \vec{k}\right) \vec{B}^{b}\left(k_{0}, \vec{k}\right)\right.
\end{aligned}
$$

$\left(E^{a, n}=-F^{a, 0 n}, B^{a, j}=-\frac{1}{2} \epsilon^{j k n} F^{a, k n}\right)$. Equations (29) and (30) are the analogs of the constitutive relations of a medium, where the "color" tensor $\kappa=\left(\kappa^{a b}\right)$ appear as generalization of the electric susceptibility. The procedure of introducing the polarization tensor may be applied to the currents $S_{\nu}$ and $R_{\nu}$ which appear due to the non-Abelian character of the group $G$. We define the non-Abelian polarization tensor

$$
Q_{\mu \nu}(x)=-Q_{\nu \mu}(x)=Q_{\mu \nu}^{a} T^{a}
$$


through the gauge covariant relation with the current $S_{\nu}$ :

$$
-S_{\nu}(x)=\mathcal{D}^{\mu}(A) Q_{\mu \nu}(x) .
$$

Related with $Q_{\mu \nu}$ are the $\mathcal{G}$-valued 3-vectors $\vec{U}$ and $\vec{V}$,

$$
U^{a, n} \equiv Q^{a, 0 n}, V^{a, n} \equiv \frac{1}{2} \epsilon^{n j l} Q^{a, j l} .
$$

The vectors $\vec{U}^{a}$ and $\vec{V}^{a}$ have no analogs in electrodynamics and will be called vectors of non-Abelian electric and magnetic polarization, respectively. We have (cf. (27), (28))

$$
\begin{aligned}
S_{0}^{a} & =(\overrightarrow{\mathcal{D}}(A) \cdot \vec{U})^{a}, \\
\vec{S}^{a} & =-\partial_{0} \vec{U}^{a}-(\overrightarrow{\mathcal{D}}(A) \times \vec{V})^{a},
\end{aligned}
$$

Due to the lack of analogy with the electrodynamical case we may assume either a linear relation between $\vec{U}^{a}, \vec{M}^{a}$, and the fields $\vec{E}^{a}, \vec{B}^{a}$, of the type

$$
\vec{U}^{a}(k)=t^{a b} \vec{E}^{b}(k), \vec{V}^{a}(k)=u^{a b}(k) \vec{B}^{b}(k) .
$$

or a non-linear (e.g. quadratic) relation among them. Here we consider the assumption of linear relation and call $t(k)$ tensor of non-Abelian electric susceptibility. The structure of the current $R_{\nu}(x)$ yields the relation

$$
R_{\nu}(x)=\mathcal{D}^{\mu}(A) * Q_{\mu \nu}(x)
$$

with the dual of the tensor $Q_{\mu \nu}$, so

$$
R_{0}^{a}=-(\overrightarrow{\mathcal{D}}(A) \cdot \vec{V})^{a}, \vec{R}^{a}=\partial_{0} \vec{V}^{a}+(\overrightarrow{\mathcal{D}}(A) \times \vec{V})^{a} .
$$

\section{In-medium Yang-Mills equations}

Inserting the expressions for the currents $J_{\nu}, R_{\nu}$ and $S_{\nu}$ in terms of the susceptibility tensors allows one to recast the averaged system of equations (15), (16) in the form

$$
\begin{aligned}
& i k^{m}\left(\delta^{a b}+g \kappa^{a b}(k)+g t^{a b}(k)\right) E^{b, m}(k)+ \\
& +g f^{a b c} \int A^{b, m}(k-q)\left(\delta^{c d}+g \kappa^{c d}(q)+g t^{a b}(q)\right) E^{d, m}(q) \frac{d^{4} q}{(2 \pi)^{4}}= \\
& =g j_{o}^{a}(k), \\
& i k_{0}\left(\delta^{a b}+g \kappa^{a b}(k)+g t^{a b}(k)\right) E^{b, m}(k)+ \\
& +\epsilon^{n m l} i k^{m}\left(\delta^{a b}-g \psi^{a b}(k)+g u^{a b}(k)\right) B^{b, l}(k)+ \\
& +\epsilon^{n m l} g f^{a b c} \int A^{b, m}(k-q)\left(\delta^{c d}-g \psi^{a b}(q)+g u^{a b}(q)\right) B^{d, l}(q) \frac{d^{4} q}{(2 \pi)^{4}}= \\
& =g j^{a, n}(k), \\
& i k^{m}\left(\delta^{a b}-g u^{a b}(k)\right) B^{b, m}(k)+
\end{aligned}
$$


In-medium Yang-Mills equations: a derivation and canonical quantization

$$
\begin{aligned}
& g f^{a b c} \int A^{b, m}(k-q)\left(\delta^{c d}-g u^{c d}(q)\right) B^{m, d}(q) \frac{d^{4} q}{(2 \pi)^{4}}=0, \\
& i k_{0}\left(\delta^{a b}-g u^{a b}(k)\right) B^{b, n}(k)+\epsilon^{n l m} i k^{l}\left(\delta^{a b}-g t^{a b}(k)\right) E^{b, m}(k)+ \\
& +g f^{a b c} \int A^{b, l}(k-q)\left(\delta^{c d}-g t^{c d}(q)\right) E^{m, d}(q) \frac{d^{4} q}{(2 \pi)^{4}}=0 .
\end{aligned}
$$

Let us define the modified "color" electric field $\vec{E}^{\prime a}$

$$
{\overrightarrow{E^{\prime}}}^{\prime a}(k) \equiv \vec{E}^{a}(k)-g \vec{U}^{a}(k)
$$

and the modified "color" magnetic field $\overrightarrow{B^{\prime a}}$

$$
\vec{B}^{\prime a}(k) \equiv \vec{B}^{a}(k)-g \vec{V}^{a}(k) .
$$

We define also the generalized electric induction

$$
\vec{D}^{a}(k) \equiv \vec{E}^{a}(k)+g \vec{P}^{a}(k)+g \vec{U}^{a}(k)
$$

and the generalized magnetic induction

$$
\vec{H}^{a}(k) \equiv \vec{B}^{a}(k)-g \vec{M}^{a}(k)+g \vec{V}^{a}(k) .
$$

From (32) one obtains

$$
\begin{aligned}
& {\overrightarrow{E^{\prime}}}^{a}(k)=\left(\delta^{a b}-g t^{a b}(k)\right) \vec{E}^{b}(k) \\
& \vec{B}^{\prime a}(k)=\left(\delta^{a b}-g u^{a b}(k)\right) \vec{B}^{b}(k)
\end{aligned}
$$

and from $(29),(30),(33)$ we get

$$
\begin{aligned}
\vec{D}^{a}(k) & =\left(\delta^{a b}+g \kappa^{a b}(k)+g t^{a b}(k)\right) \vec{E}^{b}(k), \\
\vec{H}^{a}(k) & =\left(\delta^{a b}-g \psi^{a b}(k)+g u^{a b}(k)\right) \vec{B}^{b}(k) .
\end{aligned}
$$

We write these relations in the form

$$
\begin{aligned}
& \vec{D}^{a}(k)=\epsilon^{a b}(k) \vec{E}^{b}(k) \\
& \vec{B}^{a}(k)=\mu^{a b}(k) \vec{H}^{b}(k),
\end{aligned}
$$

where

$$
\epsilon^{a b}(k) \equiv \delta^{a b}+g \kappa^{a b}(k)+g t^{a b}(k)
$$

will be called tensor of generalized electric permittivity, and

$$
\mu^{a b}(k) \equiv\left((1-g \psi+g u)^{-1}\right)^{a b}
$$

- tensor of generalized magnetic permeability. If we keep the analogy with the electromagnetic properties of the vacuum, we have to prescribe the property of 
generalized permeability $\mu_{0}$ and permittivity $\epsilon_{0}$ to the empty space with $\mu_{0}=$ const, $\epsilon_{0}=$ const. Then the definitions of $\mu$ and $\epsilon$ will read

$$
\mu^{a b} / \mu_{0} \equiv \delta^{a b}+g \chi^{a b}, \text { and } \epsilon^{a b} / \epsilon_{0} \equiv \delta^{a b}+g \kappa^{a b} .
$$

Now the system (34)-(37) written as a system of differential equations reads

$$
\begin{aligned}
& (\overrightarrow{\mathcal{D}}(A) \cdot \vec{D})^{a}=g j_{0}^{a}, \\
& (\overrightarrow{\mathcal{D}}(A) \times \vec{H})^{a}-\partial_{0} \vec{D}^{a}=g \vec{j}^{a} \\
& \left(\overrightarrow{\mathcal{D}}(A) \cdot \overrightarrow{B^{\prime}}\right)^{a}=0, \\
& \left(\overrightarrow{\mathcal{D}}(A) \times \vec{E}^{\prime}\right)^{a}-\partial_{0} \vec{B}^{\prime a}=0
\end{aligned}
$$

The system (41) together with equations (39) and (40) where the tensors $t^{a b}, u^{a b}$, $\epsilon^{a b}$ and $\mu^{a b}$ are given functions of $\left(x^{0}, \vec{x}\right)$ will be called system of in-medium Yang-Mills equations.

Our next step is to outline how relations between the susceptibility tensors and the Green's functions can be derived in principle. The Fourier transform of the system (1) written in terms of the fields $e^{c, n}=-f^{c, 0 n}$ and $b^{c, n}=-\frac{1}{2} \epsilon^{n j m} f^{c, j m}$, $(c=1, \ldots, r ; n=1,2,3)$ and restricted to terms linear in the external field gives for the external current

$$
\begin{aligned}
& g j_{0}^{a}(k)=i \vec{k} \cdot \vec{e}^{a}(k) \\
& g \vec{j}^{a}(k)=i \frac{k^{2}}{k_{0}} \vec{e}^{a}(k)+i \frac{\vec{k} \cdot \vec{e}^{a}(k)}{k_{0}} \vec{k}
\end{aligned}
$$

$\left(k=\left(k_{0}, \vec{k}\right), k^{2}=k_{0}^{2}-\vec{k}^{2}\right)$. The substitution of (42) into the r.h.s of the system of equations (34)- (37) gives a non-linear system for the fields $\vec{E}^{a}(k)$ and $\vec{B}^{a}(k)$ (recall that $\vec{E}^{a}(k)=i k_{0} \vec{A}^{a}(k)$ in the gauge $\left.\hat{A}_{0}=0\right)$. In the linear approximation with respect to the external field we have to keep only the linear terms in the expressions for $\vec{E}^{a}$ and $\vec{B}^{a}$

$$
\vec{E}^{a}(k)=\phi^{a b} \bar{e}^{-b}(k), \vec{B}^{a}(k)=\theta^{a b} \bar{e}^{-b}(k) .
$$

Here $\phi^{a b}$ and $\theta^{a b}$ depend on $k$ as well as on the tensors $\kappa, \psi, t$ and $u$. The form (43) implies that $\left[A_{\mu}, A_{\nu}\right]$ and $\left[A_{\mu}, F_{\sigma \tau}\right]$ in equations (18), (19) are quadratic in the external field and have to be dropped out thus simplifying the currents $S_{\nu}$ and $R_{\nu}$ to

$$
\begin{aligned}
S_{\nu}^{a}(x) & =i\left\{\partial^{\mu}<\left[\hat{A}_{\mu}(x), \hat{A}_{\nu}(x)\right]^{a}>+<\left[\hat{A}^{\mu}(x), \hat{F}_{\mu \nu}(x)\right]^{a}>\right\} \\
& =g \int K_{\nu \lambda}^{a b}(x-y) a^{b, \lambda}(y) d^{4} y
\end{aligned}
$$

and

$$
\begin{aligned}
R_{\nu}^{a}(x) & =\frac{i}{2} \epsilon_{\nu \mu \sigma \tau}\left\{\partial^{\mu}<\left[\hat{A}^{\sigma}(x), \hat{A}^{\tau}(x)\right]^{a}>+<\left[\hat{A}^{\mu}(x), \hat{F}^{\sigma \tau}(x)\right]^{a}>\right\} \\
& =g \int L_{\nu \lambda}^{a b}(x-y) a^{b, \lambda}(y) d^{4} y .
\end{aligned}
$$


Adding the formula for $J_{\nu}(x)$,

$$
J_{\nu}^{a}(x)=<\hat{I}_{\nu}^{a}(x)>=g \int G_{\nu \lambda}^{a b}(x-y) a^{b, \lambda}(y) d^{4} y
$$

one may express the three currents (44), (45), (46), further in terms of the external field since in any fixed gauge for $a^{b, \lambda}$ the equations

$$
\begin{aligned}
& \vec{e}^{c}(k)=i k_{0} \vec{a}^{c}(k)-i \vec{k} a^{0, c}(k) \\
& \vec{b}^{c}(k)=\frac{1}{k_{0}} \vec{k} \times \vec{e}^{c}(k)=i \vec{k} \times \vec{a}^{c}(k),
\end{aligned}
$$

determine $a^{c, \lambda}$ in terms of $\bar{e}^{t}$.

On the other hand, the currents $J_{\nu}$ and $S_{\nu}$ are used to introduce the generalized susceptibility tensors via (26) and (31). The assumption that these tensors are linearly connected with $\vec{E}^{a}$ and $\vec{B}^{a}$ (equations (29), (30) and (32)) allows one to express them linearly by $\vec{e}^{b}$ using (43). The obtained two expressions for each of the currents $J_{\nu}$ and $S_{\nu}$ allow one to derive algebraic relations among the tensors $\kappa, \psi, t$ and $u$, and the Green's functions $G_{\nu \lambda}^{a b}, K_{\nu \lambda}^{a b}$.

Let us note that if the tensors $t^{a b}$ and $u^{a b}$ depend in a non-linear (e.g. quadratic) way on the fields $\vec{E}^{a}$ and $\vec{B}^{a}$, then they have no contribution in the averaged system (34) - (37) in the approximation linear in the external field. Then the modified fields $\vec{E}^{\prime a}$ and $\vec{B}^{\prime a}$ reduce to $\vec{E}^{a}$ and $\vec{B}^{a}$, respectively, and the in-medium Yang-Mills equations become

$$
\begin{aligned}
& (\overrightarrow{\mathcal{D}}(A) \cdot \vec{D})^{a}=g j_{0}^{a}, \\
& (\overrightarrow{\mathcal{D}}(A) \times \vec{H})^{a}-\partial_{0} \vec{D}^{a}=g \vec{j}^{a}, \\
& (\overrightarrow{\mathcal{D}}(A) \cdot \vec{B})^{a}=0, \\
& (\overrightarrow{\mathcal{D}}(A) \times \vec{E})^{a}-\partial_{0} \vec{B}^{a}=0 .
\end{aligned}
$$

\section{Approximation up to second order in $\mathrm{g}$}

Considered as perturbative series in $g$ both Green's functions $K_{\nu \lambda}^{a b}$ and $L_{\nu \lambda}^{a b}$ have their first non-vanishing term of first order in $g$. Indeed, let us take a perturbative expansion of $\hat{A}_{\mu}(x)$ and $\hat{F}_{\mu \nu}(x)$ in powers of $g$ beginning with the free field operators $\hat{A}_{(0) \mu}$ and $\hat{F}_{(0) \mu \nu}$, respectively,

$$
\begin{aligned}
& \hat{A}_{\mu}(x)=\hat{A}_{(0) \mu}(x)+\sum_{n \geq 1} g^{n} \hat{A}_{(n) \mu}(x) \\
& \hat{F}_{\mu \nu}(x)=\hat{F}_{(0) \mu \nu}(x)+\sum_{n \geq 1} g^{n} \hat{F}_{(n) \mu \nu}(x)
\end{aligned}
$$


$\left(\hat{A}_{(n) \mu}(x)\right.$ being the $n-t h$ approximation). Then the observation that $\hat{A}_{(0) \mu}(x)$ and $\hat{F}_{(0) \mu \nu}(x)$ commute with the current $\hat{I}_{\lambda}$ expressed in terms of free spinor operators yields the conclusion that both $K_{\nu \lambda}^{a b}$ and $L_{\nu \lambda}^{a b}$ are of order $g$. According to (44) and (45) the terms with $g S_{\nu}$ and $g R_{\nu}$ in the r.h.s of the system of equations (15), (16), are of third order in $g$ while the term with $g J_{\nu}$ is of second order (like the case of electrodynamics).

In this Section we consider the systems (15), (16), up to second order in $g$ (in the approximation linear w.r.t. the external field). In this approximation the currents $g S_{\nu}$ and $g R_{\nu}$ drop out, the covariant derivatives in the l.h.s of (15), (16) reduce to simple partial derivatives because $\left[A^{\mu}, F_{\sigma \tau}\right]$ are quadratic in the external field due to equation (43), and we obtain the simplified form

$$
\begin{aligned}
& \partial^{\mu} \tilde{F}_{\mu \nu}^{a}=g J_{\nu}^{a}+g j_{\nu}^{a}, \\
& \partial^{\mu} * \tilde{F}_{\mu \nu}^{a}=0, \\
& \tilde{F}_{\mu \nu}^{a}=\partial_{\mu} A_{\nu}^{a}-\partial_{\nu} A_{\mu}^{a} .
\end{aligned}
$$

Local commutativity of the currents $J_{\nu}^{a}(x)$ gives that

$$
\partial^{\mu} G_{\mu \nu}^{a b}(x)=0=\partial^{\nu} G_{\mu \nu}^{a b}(x)
$$

because $\partial^{\mu} J_{\mu}(x)=0$ in our approximation. Using the Fourier transform of the last equality and the system (47) (in the gauge $\partial_{\lambda} a^{\lambda}=0$ ) we recast equation (46).

$$
J_{\nu}^{a}(k)=g G_{\nu \lambda}^{a b}(k) a^{\lambda, b}(k)
$$

in the form $\left(k=\left(k_{0}, \vec{k}\right)\right)$

$$
\begin{aligned}
& J_{0}^{a}\left(k_{0}, \vec{k}\right)=i g \frac{k^{m}}{k_{0}^{2}} G_{m l}^{a b}\left(k_{0}, \vec{k}\right) e^{b, l}\left(k_{0}, \vec{k}\right), \\
& J_{n}^{a}\left(k_{0}, \vec{k}\right)=-i g \frac{1}{k_{0}} G_{n l}^{a b}\left(k_{0}, \vec{k}\right) e^{b, l}\left(k_{0}, \vec{k}\right) .
\end{aligned}
$$

For a homogeneous and isotropic medium $G_{m n}^{a b}\left(k_{0}, \vec{k}\right)$ may be written as a sum of tensors

$$
G_{m n}^{a b}\left(k_{0}, \vec{k}\right)=-i k_{0}\left[\tilde{\sigma}_{l}^{a b}(k) \frac{k_{m} k_{n}}{\vec{k}^{2}}+\tilde{\sigma}_{t}^{a b}(k)\left(\delta_{n m}-\frac{k_{m} k_{n}}{\vec{k}^{2}}\right)\right]
$$

where $\tilde{\sigma}_{l}^{a b}$ and $\tilde{\sigma}_{t}^{a b}$ are "color" tensors depending on $k=|\vec{k}|$ and arise as analogs of the so called exterior longitudinal and transverse electric conductivity coefficients [31]. This form of $G_{m n}^{a b}$ generates a representation of $J_{0}^{a}$ and $J_{n}^{a}$,

$$
\begin{aligned}
& J_{0}^{a}\left(k_{0}, \vec{k}\right)=\frac{g}{k_{0}} \tilde{\sigma}_{l}^{a b}\left(\vec{k} \cdot \vec{e}^{b}\right) \\
& J_{n}^{a}\left(k_{0}, \vec{k}\right)=g \tilde{\sigma}_{l}^{a b}(k) \frac{\vec{k} \cdot \vec{e}^{b}}{\vec{k}^{2}} k_{n}+g \tilde{\sigma}_{t}^{a b}(k) \frac{\left(\left(\vec{k} \times \vec{e}^{b}\right) \times \vec{k}\right)_{n}}{\vec{k}^{2}},
\end{aligned}
$$


the second equation being a decomposition of $J_{n}^{a}$ into longitudinal and transverse parts with respect to $\vec{k}$. It is convenient to write the vectors $\vec{P}^{a}$ and $\vec{M}^{a}$, equations (29), (30), in the form

$$
\begin{aligned}
& \vec{P}^{a}\left(k_{0}, \vec{k}\right)=\kappa^{a b}\left(k_{0}, \vec{k}\right) \vec{E}^{b}\left(k_{0}, \vec{k}\right), \\
& \vec{M}^{a}\left(k_{0}, \vec{k}\right)=\left(\mu^{-1}\right)^{a b}\left(k_{0}, \vec{k}\right) \chi^{b c}\left(k_{0}, \vec{k}\right) \vec{B}^{c}\left(k_{0}, \vec{k}\right),
\end{aligned}
$$

(i.e. we write $\psi=\mu^{-1} \cdot \chi$ ) where $\mu^{a b}=\delta^{a b}+g \chi^{a b}$. The Fourier transform of the system (6) written in terms of $E^{e, n}=-\tilde{F}^{a, 0 n}$ and $B^{a, n}=-\frac{1}{2} \epsilon^{n m l} \tilde{F}^{a, m l}$ (i.e. the system of equations (34) - (37) in the approximation considered) reads

$$
\begin{aligned}
& i k^{m}\left(\delta^{a b}+\kappa^{a b}\left(k_{0}, \vec{k}\right)\right) E^{b, m}\left(k_{0}, \vec{k}\right)=g j_{0}^{a}\left(k_{0}, \vec{k}\right), \\
& i k_{0}\left(\delta^{a b}+\kappa^{a b}\left(k_{0}, \vec{k}\right) E^{b, m}\left(k_{0}, \vec{k}\right)+\right. \\
& \epsilon^{n m l} i k^{m}\left(\delta^{a b}-g\left(\mu^{-1}\right)^{a b}\left(k_{0}, \vec{k}\right) \chi^{b c}\left(k_{0}, \vec{k}\right)\right) B^{b, l}\left(k_{0}, \vec{k}\right)=g j^{a, n}\left(k_{0}, \vec{k}\right), \\
& i k^{m} B^{a, m}\left(k_{0}, \vec{k}\right)=0, \\
& i k_{0} B^{a, n}\left(k_{0}, \vec{k}\right)+\epsilon^{n m l} i k^{l} E^{a, m}\left(k_{0}, \vec{k}\right)=0 .
\end{aligned}
$$

The vectors of generalized electric and magnetic induction are

$$
\begin{aligned}
\vec{D}^{a} & =\vec{E}^{a}+g \vec{P}^{a}=\epsilon^{a b} \vec{E}^{b}, \\
\vec{H}^{a} & =\vec{B}^{a}-g \vec{M}^{a}=\left(\mu^{-1}\right)^{a b} \vec{B}^{b},
\end{aligned}
$$

with $\epsilon^{a b}=\delta^{a b}+g \kappa^{a b}$. In terms of these fields the form of the system (51) as a system of differential equations reads

$$
\begin{aligned}
& \operatorname{div} \vec{D}^{a}=j_{0}^{a}, \\
& \operatorname{rot} \vec{H}^{a}-\partial_{0} \vec{D}^{a}=g \vec{j}^{a}, \\
& \operatorname{div} \vec{B}^{a}=0, \\
& \operatorname{rot} \vec{E}^{a}-\partial_{0} \vec{B}^{a}=0 .
\end{aligned}
$$

It follows from the above equations that

$$
\begin{aligned}
& \vec{D}_{\|}^{c}\left(k_{0}, \vec{k}\right) \equiv \frac{1}{\vec{k}^{2}}\left(\vec{k} \cdot \vec{D}^{c}\left(k_{0}, \vec{k}\right)\right) \vec{k}=\epsilon^{c d}\left(k_{0}, \vec{k}\right) \vec{E}_{\|}^{d}\left(k_{0}, \vec{k}\right)=\vec{e}_{\|}^{c}\left(k_{0}, \vec{k}\right), \\
& {\left[k_{0}^{2} \epsilon^{a b}-\vec{k}^{2}\left(\mu^{-1}\right)^{a b}\right] \vec{E}_{\perp}^{b}\left(k_{0}, \vec{k}\right)=\left(k_{0}^{2}-\vec{k}^{2}\right) \vec{e}_{\perp}^{a}\left(k_{0}, \vec{k}\right)}
\end{aligned}
$$

and

$$
\left[k_{0}^{2} \epsilon^{a b}-\vec{k}^{2}\left(\mu^{-1}\right)^{a b}\right] \vec{B}_{\perp}^{b}\left(k_{0}, \vec{k}\right)=\left(k_{0}^{2}-\vec{k}^{2}\right) \vec{h}^{a}\left(k_{0}, \vec{k}\right)
$$


with $\vec{B}_{\|}^{a}=0$, where for a vector $\vec{v}^{a}$ we define

$$
\vec{v}_{\perp}^{a}=\frac{1}{\vec{k}^{2}}\left(\vec{k} \times \vec{v}^{a}\right) \times \vec{k}
$$

With the help of equations (49), (50), and the relations

$$
\begin{aligned}
J_{0}^{a}\left(k_{0} \vec{k}\right) & =-i \vec{k} \cdot \vec{P}^{a}\left(k_{0}, \vec{k}\right), \\
\vec{J}^{a}\left(k_{0} \vec{k}\right) & =-i k_{0} \vec{P}^{a}\left(k_{0} \vec{k}\right)+i \vec{k} \times \vec{M}^{a}\left(k_{0} \vec{k}\right),
\end{aligned}
$$

now we derive

$$
k_{0} \kappa^{a b}\left(k_{0} \vec{k}\right)=i g \tilde{\sigma}_{t}^{a b}(|\vec{k}|) \epsilon^{c b}\left(k_{0} \vec{k}\right)
$$

or, in a compact matrix notation

$$
1-\epsilon^{-1}=i g^{2} \tilde{\sigma}_{t}
$$

as well as the relation

$$
\begin{aligned}
& 1-\mu^{-1}=g \mu^{-1} \chi= \\
& i \frac{k_{0}}{\vec{k}^{2}} g^{2}\left\{\tilde{\sigma}_{t}\left(1-i g^{2} k_{0} \frac{\tilde{\sigma}_{t}}{k_{0}^{2}-\vec{k}^{2}}\right)^{-1}-\left(1-i \frac{g^{2}}{k_{0}} \tilde{\sigma}_{l}\right)^{-1} \tilde{\sigma}_{l}\right\} .
\end{aligned}
$$

Equations (53) and (54) resemble their counterparts in electrodynamics and by analogy, we call

$$
\begin{aligned}
& \sigma_{l}=\left(1-i \frac{g^{2}}{k_{0}} \tilde{\sigma}_{l}\right)^{-1} \tilde{\sigma}_{l}, \\
& \sigma_{t}=\tilde{\sigma}_{t}\left(1-i g^{2} k_{0} \frac{\tilde{\sigma}_{t}}{k_{0}^{2}-\vec{k}^{2}}\right)^{-1},
\end{aligned}
$$

tensors of intrinsic longitudinal and transverse conductivity, respectively.

\section{Lagrangian and canonical quantization}

In this section we consider the case when the tensors $t^{a b}$ and $u^{a b}$, equation (32), may be neglected, so the in-medium Yang-Mills fields are described by the system (48).

For a medium in rest with respect to a fixed Lorenz frame we define the antisymmetric tensors $H^{a, \mu \nu}$ by

$$
\begin{aligned}
& H^{a, 0 \kappa}=-D^{a, \kappa}, \\
& H^{a, m n}=-\epsilon^{m n k} H^{a, k}, n, m=1,2,3 .
\end{aligned}
$$

The components of the tensors (55) read

$$
H^{a, \mu \nu}=\left(\mu^{-1}\right)^{a b} F^{b, \mu \nu}+\left(\epsilon-\mu^{-1}\right)^{a b} V^{\tau}\left(g^{\mu \sigma} V^{\nu}-g^{\nu \sigma} V^{\mu}\right) F_{\sigma \tau}^{b},
$$


if the medium moves uniformly with velocity $V^{\mu}\left(V^{2}=1\right)$. For vanishing exterior current $j_{\mu}^{a}$ the Lagrangian

$$
\mathcal{L}_{0}=-\frac{1}{4} F_{\mu \nu}^{a} H^{a, \mu \nu}
$$

reproduces the first two equations of the system (52) written in a covariant form

$$
D_{\mu}(A) H^{a, \mu \nu}=0,
$$

valid for any reference frame. Since in (56) and (57) the tensor $F_{\mu \nu}^{a}$ reads

$$
F_{\mu \nu}^{a}=\partial_{\mu} A_{\nu}^{a}-\partial_{\nu} A_{\mu}^{a}-g f^{a b c} A_{\mu}^{b} A_{\nu}^{c}
$$

it satisfies the covariant form of the last two equations of the system (52), i.e.

$$
D_{\lambda}(A) F_{\mu \nu}^{a}+D_{\mu}(A) F_{\nu \lambda}^{a}+D_{\nu}(A) F_{\lambda \mu}^{a}=0 .
$$

In order to perform the canonical quantization, we add a gauge fixing term $\mathcal{L}_{g f}$ to $\mathcal{L}_{0}$,

$$
\mathcal{L}_{g f}=\frac{\xi}{2}\left(B^{a} B^{a}\right)-B^{a}\left(\partial^{\mu} A_{\mu}^{a}+\left(n^{2}-1\right)^{a b} V^{\mu} \partial_{\mu} V^{\lambda} A_{\lambda}^{b}\right),
$$

where $\xi$ is a real parameter and $B^{a}(a=1, \ldots, r)$ are real auxiliary scalar fields. The tensor $\left(n^{2}\right)^{a b}=\epsilon^{a c} \mu^{c b}$ is the generalization of the classical refraction coefficient. The form $(58)$ of $\mathcal{L}_{g f}$ is taken in analogy with the case of electromagnetic field in a medium [32]. In the sequel we will consider the case $\epsilon^{a b}=\epsilon \delta^{a b}, \mu^{a b}=\mu \delta^{a b}$, where $\epsilon$ and $\mu$ are constants. Then $\left(n^{2}\right)^{a b}=\epsilon \mu \delta^{a b}$ and the notation $\beta=n^{2}-1$ will be used (here and below we shall write $n^{2}$ for $\left.\epsilon \mu\right)$. We split the total Lagrangian $\mathcal{L}=\mathcal{L}_{0}+\mathcal{L}_{\text {gf }}$ into two parts, $\mathcal{L}=\mathcal{L}_{\text {free }}+\mathcal{L}_{\text {int }}$, where $\mathcal{L}_{\text {int }}$ contains all terms containing the gauge coupling constant $g$. The part $\mathcal{L}_{\text {free }}$ equals

$$
\mathcal{L}_{\text {free }}=-\frac{1}{4} \tilde{F}_{\mu \nu}^{a} \tilde{H}^{a, \mu \nu}+\mathcal{L}_{g f}
$$

where

$$
\tilde{F}_{\lambda \mu}^{a}=\partial_{\lambda} A_{\mu}^{a}-\partial_{\mu} A_{\lambda}^{a}
$$

and

$$
\tilde{H}^{a, \mu \nu}=\left(\mu^{-1}\right)^{a b} \tilde{F}^{b, \mu \nu}+\left(\epsilon-\mu^{-1}\right)^{a b} V^{\tau}\left(g^{\mu \sigma} V^{\nu}-g^{\nu \sigma} V^{\mu}\right) \tilde{F}_{\sigma \tau}^{b} .
$$

The equations of motion following from the free field part $\mathcal{L}_{\text {free }}$ are

$$
\begin{aligned}
& \square A_{\lambda}^{a}+\beta(V \cdot \partial)^{2} A_{\lambda}^{a}+(\mu-\xi) \partial_{\lambda} B^{a} \equiv Q A_{\lambda}^{a}+(\mu-\xi) \partial_{\lambda} B^{a}=0, \\
& \square B^{a}+\beta(V \cdot \partial)^{2} B^{a} \equiv Q B^{a}=0, \\
& \xi B^{a}=\partial \cdot A^{a}+\beta(V \cdot \partial)\left(V \cdot A^{a}\right),
\end{aligned}
$$

where $Q=\square+\beta(V \cdot \partial)^{2}$. We choose $A_{\lambda}^{a}(x)$ as generalized coordinates, the conjugate momenta being

$$
\pi^{a, \mu}(x)=\frac{\partial \mathcal{L}_{0}}{\partial\left(\partial_{0} A_{\mu}^{a}\right)}(x),
$$


or

$$
\begin{aligned}
& \pi^{a, 0}=-\left[1+\beta V_{0}^{2}\right] B^{a}, \\
& \pi^{a, k}=\beta V_{0} V_{k} B^{a}+\frac{\beta}{\mu} V_{0} V_{n} \tilde{F}_{k n}^{a}+\left[\left(1+2 \beta V_{0}^{2}\right) \delta_{k n}-\beta V_{k} V_{n}\right] \tilde{F}_{0 n},
\end{aligned}
$$

$(k=1,2,3)$. From the equal time canonical commutation relations

$$
\begin{aligned}
& {\left[A_{\lambda}(x), A_{\mu}(y)\right]_{x_{0}=y_{0}}=0} \\
& {\left[\pi^{a, \lambda}(x), \pi^{b, \mu}(y)\right]_{x_{0}=y_{0}}=0,} \\
& {\left[\pi^{a, \lambda}(x), A_{\mu}^{b}(y)\right]_{x_{0}=y_{0}}=-i \delta^{a b} \delta_{\mu}^{\lambda} \delta(\vec{x}-\vec{y}),}
\end{aligned}
$$

one derives

$$
\begin{aligned}
{\left[B^{a}(x), A_{\lambda}^{b}(y)\right]_{x_{0}=y_{0}} } & =\frac{i \delta^{a b} \delta_{0 \lambda}}{\left(1+\beta V_{0}^{2}\right)} \delta(\vec{x}-\vec{y}) \\
{\left[B^{a}(x), \partial_{0} A_{n}^{b}(y)\right]_{x_{0}=y_{0}} } & =\frac{-i \delta^{a b}}{\left(1+\beta V_{0}^{2}\right)} \frac{\partial}{\partial x^{n}} \delta(\vec{x}-\vec{y})
\end{aligned}
$$

as well as

$$
\begin{aligned}
& {\left[\partial_{0} A_{0}^{a}(x), A_{0}^{b}(y)\right]_{x_{0}=y_{0}}=\frac{i \delta^{a b} \delta(\vec{x}-\vec{y})}{\left(1+\beta V_{0}^{2}\right)^{2}}\left[\xi-\mu \beta^{2} \frac{V_{0}^{2} \vec{V}^{2}}{(1+\alpha)}\right],} \\
& {\left[\partial_{0} A_{0}^{a}(x), A_{n}^{b}(y)\right]_{x_{0}=y_{0}}=-\frac{i \mu \beta V_{0} V_{n} \delta^{a b}}{(1+\beta)\left(1+\beta V_{0}^{2}\right)} \delta(\vec{x}-\vec{y}),} \\
& {\left[\partial_{0} B^{a}(x), A_{0}^{b}(y)\right]_{x_{0}=y_{0}}=2 i \frac{\beta V_{0}}{\left(1+\beta V_{0}^{2}\right)^{2}} \delta^{a b}\left(V_{n} \frac{\partial}{\partial x^{n}}\right) \delta(\vec{x}-\vec{y}),} \\
& {\left[\partial_{0} B^{a}(x), A_{n}^{b}(y)\right]_{x_{0}=y_{0}}=\frac{i \delta^{a b}}{\left(1+\beta V_{0}^{2}\right)} \frac{\partial}{\partial x^{n}} \delta(\vec{x}-\vec{y}) .}
\end{aligned}
$$

Following [32] we introduce the tensor

$$
b_{\tau}^{\sigma}=\delta_{\tau}^{\sigma}+(n-1) V^{\sigma} V_{\tau},
$$

satisfying

$$
\left(b^{l}\right)_{\tau}^{\sigma}=\delta_{\tau}^{\sigma}+\left(n^{l}-1\right) V^{\sigma} V_{\tau}
$$

for every integer number $l$. In terms of $b_{\tau}^{\sigma}$, the gauge fixing term $\mathcal{L}_{g f}$ reads

$$
\mathcal{L}_{g f}=\frac{\xi}{2} B^{a} B^{a}-B^{a} \partial^{\mu}\left(b^{2}\right)_{\mu}^{\tau} A_{\tau}
$$

and the operator $\mathrm{Q}$ takes the form

$$
Q=\partial^{\mu}\left(b^{2}\right)_{\mu}^{\tau} \partial_{\tau}
$$

\section{Commutation relations at non-equal times}

We define the functions

$$
F(x) \equiv i \int \frac{d^{4} k}{(2 \pi)^{3}} \exp (-i k \cdot x) \delta(Q(k)) \epsilon(V \cdot k)
$$


In-medium Yang-Mills equations: a derivation and canonical quantization

and

$$
H(x) \equiv i \int \frac{d^{4} k}{(2 \pi)^{3}} \exp (-i k \cdot x) \delta^{\prime}(Q(k)) \epsilon(V \cdot k),
$$

with the notations

$$
Q(k) \equiv k^{2}+\beta(V \cdot k)^{2}=\left(1+\beta V_{0}^{2}\right)\left(k_{0}-k_{+}(k)\right)\left(k_{0}-k_{-}(k)\right)
$$

and

$$
k_{ \pm}(\vec{k}) \equiv \frac{1}{\left(1+\beta V_{0}^{2}\right)}\left[\beta V_{0}(\vec{k} \cdot \vec{V}) \pm \sqrt{\left(1+\beta V_{0}^{2}\right) \vec{k}^{2}-\beta(\vec{k} \cdot \vec{V})^{2}}\right]
$$

Note that $k_{+}(\vec{k})$ is negative for reference frames in which $\beta \overrightarrow{V^{2}}>1$. However, it always holds that $k_{ \pm}(\vec{k})=-k_{\mp}(-\overrightarrow{-k})$.

The functions $F$ and $H$ have the properties $Q F=0, Q H=F$, as well as

$$
\begin{aligned}
& \partial_{0} F(0, \vec{x})=\frac{1}{\left(1+\beta V_{0}^{2}\right)} \delta(\vec{x}), \\
& F(0, \vec{x})=0, \\
& H(0, \vec{x})=\partial_{0} H(0, \vec{x})=\partial_{0}^{2} H(0, \vec{x})=0, \\
& \partial_{0}^{3} H(0, \vec{x})=\frac{1}{\left(1+\beta V_{0}^{2}\right)^{2}} \delta(\vec{x}) .
\end{aligned}
$$

Equations (65) and (67) yield

$$
F(x)=\frac{i}{(2 \pi)^{3}} \int \frac{d^{3} k}{\left(1+\beta V_{0}^{2}\right)} \frac{1}{\left|k_{+}(\vec{k})-k_{-}(\vec{k})\right|}\left(e^{-i k^{\prime} x}-e^{-i k^{\prime \prime} x}\right),
$$

where $k^{\prime}=\left(k_{+}(\vec{k}), \vec{k}\right), k^{\prime \prime}=\left(k_{-}(\vec{k}), \vec{k}\right)$. The current

$$
J_{\mu}(y, x)=F(y-x) \mathcal{D}^{\leftrightarrow}{ }_{x, \mu} g(x)+H(y-x) \mathcal{D}^{\leftrightarrow}{ }_{x, \mu} Q g(x)
$$

is conserved, $\frac{\partial}{\partial x^{\mu}} J^{\mu}(y, x)=0$, for every solution $g(x)$ of

$$
Q^{2} g(x)=0 \text {. }
$$

The operator $\mathcal{D}_{x, \mu}$ is defined as

$$
\mathcal{D}_{x, \mu} \equiv \frac{\partial}{\partial x^{\mu}}+\beta V_{\mu}\left(V^{\lambda} \frac{\partial}{\partial x^{\lambda}}\right)
$$

and

$$
A \mathcal{D}^{\leftrightarrow}{ }_{x, \mu} B \equiv A\left(\mathcal{D}_{x, \mu} B\right)-\left(\mathcal{D}_{x, \mu} A\right) B .
$$

Therefore the integral

$$
\int_{\Sigma} d \sigma^{\mu}(z) J_{\mu}(y, z)
$$

$\left(d \sigma^{\mu}(z)\right.$ is the element on the surface $\left.\Sigma\right)$ depends only on $y$. For a space-like $\Sigma$ with $d \sigma^{\mu}(z)=(1,0,0,0) d^{3} z$ we define

$$
G(y) \equiv \int_{z^{0}=y^{0}} d^{3} z J_{0}(y, z) .
$$


It follows from equation (71) and the properties (68) - (69) that

$$
G(y)=g(y)=\int_{z^{0}=y^{0}} d^{3} z\left[F(y-z) \mathcal{D}_{z, 0}^{\leftrightarrow} g(z)+H(y-z) \mathcal{D}_{z, 0}^{\leftrightarrow} Q g(z)\right] .(74
$$

From (62) and (63) one derives

$$
Q^{2} A_{\lambda}^{a}(x)=0 .
$$

Therefore we obtain

$$
\left.A_{\lambda}^{a}(x)=\int_{x^{0}=z^{0}} d^{3} z\left[F(x-z) \mathcal{D}^{\leftrightarrow}{ }_{z, 0} A_{\lambda}^{a}(z)+H(x-z)\right) \mathcal{D}^{\leftrightarrow}{ }_{z, 0} Q A_{\lambda}^{a}(z)\right] .
$$

This expression allows one to calculate the commutators among the fields $A_{\lambda}^{a}(x)$ for arbitrary times using the equal time commutators appearing in the r.h.s of the identify

$$
\begin{aligned}
{\left[A_{\lambda}^{a}(x), A_{\mu}^{b}(y)\right]=} & \int_{z_{0}=y_{0}} d^{3} z\left\{F(x-z) \mathcal{D}_{z, 0}^{\leftrightarrow}\left[A_{\lambda}^{a}(z), A_{\mu}^{b}(y)\right]\right. \\
& \left.+H(x-z) \mathcal{D}_{z, 0}^{\leftrightarrow}(\xi-\mu) \frac{\partial}{\partial z^{\lambda}}\left[B^{a}(z), A_{\mu}^{b}(y)\right]\right\} .
\end{aligned}
$$

The result reads

$$
\left[A_{\lambda}^{a}(x), A_{\mu}^{b}(y)\right]=i \mu \delta^{a b}\left(g_{\lambda \mu}-\frac{\beta}{1+\beta} V_{\lambda} V_{\mu}\right) F(x-y)-i(\mu-\xi) \delta^{a b} \frac{\partial^{2} H(x-y)}{\partial x^{\lambda} \partial x^{\mu}} .
$$

Using equation (64), i.e.

$$
\xi B^{a}(x)=\mathcal{D}_{x, \mu} A^{a, \mu}(x),
$$

with $\mathcal{D}_{x, \mu}$ given by $(72)$, one obtains from equation (77)

$$
\left[A_{\lambda}^{a}(x), B^{b}(y)\right]=-i \delta^{a b} \frac{\partial}{\partial x^{\lambda}} F(x-y)
$$

and

$$
\left[B^{a}(x), B^{b}(y)\right]=0 .
$$

The commutation relations (77-79) generalize those derived for the electromagnetic filed in a medium [32] in a natural way.

One may use the technique of [32] to derive the commutation relations (77) in a much simpler way. Indeed, the change of variables

$$
\begin{aligned}
& x^{\lambda} \quad \rightarrow y^{\lambda}=\left(b^{-1}\right)_{\mu}^{\lambda} x^{\mu}, \\
& A_{\lambda}^{a}(x) \rightarrow \Gamma_{\lambda}^{a}(y)=\frac{1}{\sqrt{\mu}} A_{\sigma}^{a}(x) b_{\lambda}^{\sigma}, \\
& B^{a}(x) \rightarrow \Delta^{a}(y)=\sqrt{\mu} B^{a}(x)
\end{aligned}
$$

and

$$
g \rightarrow g^{\prime}=\sqrt{\mu} g, \xi \rightarrow \xi^{\prime}=\xi / \mu,
$$

brings the Lagrangian

$\mathcal{L}=-\frac{1}{4} F^{a, \mu \nu}(x) \mathcal{H}_{\mu \nu}^{a}(x)-\xi B^{a}(x)\left[\partial \cdot A^{a}(x)+\left(n^{2}-1\right)(V \cdot \partial) V \cdot A^{a}(x)\right]+\frac{1}{2} B^{a}(x) B^{a}(x)$ 
to the form

$$
\mathcal{L}=-\frac{1}{4} G^{a, \mu \nu}(y) G_{\mu \nu}^{a}(y)-\xi^{\prime} \Delta^{a}(y) \frac{\partial}{\partial y^{\mu}} \Gamma^{a, \mu}(y)+\frac{1}{2} \Delta^{a}(y) \Delta^{a}(y),
$$

where

$$
G_{\mu \nu}^{a}(y)=\frac{\partial \Gamma_{\nu}^{a}(y)}{\partial y^{\mu}}-\frac{\partial \Gamma_{\mu}^{a}(y)}{\partial y^{\nu}}-g^{\prime} f^{a b c} \Gamma_{\mu}^{b}(y) \Gamma_{\nu}^{c}(y)
$$

In the approximation where one takes $g^{\prime} \rightarrow 0$ this Lagrangian describes a system of $r=\operatorname{dim}(G)$ non-interacting $U(1)$-gauge fields, i.e. a system of $r$ free "electromagnetic" fields with the corresponding gauge fixing terms containing the fields $\Delta^{a}$. After quantization of each type $\Gamma_{\mu}^{a}(x),(a=1, \ldots, r)$, of "electromagnetic" fields one obtains the commutation relations [32]

$$
\left[\Gamma_{\lambda}^{a}(y), \Gamma_{\nu}^{b}\left(y^{\prime}\right)\right]=i g_{\lambda \nu} \delta^{a b} \mathcal{D}_{0}\left(y-y^{\prime}\right)-i\left(1-\xi^{\prime}\right) \frac{\partial^{2}}{\partial y^{\lambda} \partial y^{\nu}} E\left(y-y^{\prime}\right),
$$

where

$$
\begin{aligned}
& D_{0}(y)=i \int \frac{d^{4} k}{(2 \pi)^{3}} e^{-i k y} \epsilon\left(k_{0}\right) \delta\left(k^{2}\right), \\
& E(y)=i \int \frac{d^{4} k}{(2 \pi)^{3}} e^{-i k y} \epsilon\left(k_{0}\right) \delta^{\prime}\left(k^{2}\right) .
\end{aligned}
$$

Going back to the fields $A_{\lambda}^{a}$ and the variables $\left(x^{\mu}\right)$, one recovers equation (77) having in mind that $D_{0}\left(b^{-1} \cdot x\right)=F(x)$, and $E\left(b^{-1} \cdot x\right)=H(x)$.

\section{Physical subspace of the Fock space}

We introduce the Fourier integral for the fields $A_{\lambda}^{d}(x)$ and $B^{a}(x)$,

$$
\begin{aligned}
& A_{\lambda}^{d}(x)=\frac{1}{(2 \pi)^{3 / 2}} \int d^{4} p \theta\left(p_{0}\right)\left[e^{i p x} a_{\lambda}^{+d}(p)+e^{-i p x} a_{\lambda}^{-d}(p)\right], \\
& B^{a}(x)=\frac{1}{(2 \pi)^{3 / 2}} \int d^{4} p \theta\left(p_{0}\right)\left[e^{i p x} b^{+a}(p)+e^{-i p x} b^{-a}(p)\right] .
\end{aligned}
$$

As a consequence of the equations of motion one obtains

$$
\begin{aligned}
& \theta\left(k^{0}\right)\left[Q(k) a_{\lambda}^{ \pm d}(k) \pm i(\mu-\xi) b^{ \pm d}(k)\right]=0, \\
& \theta\left(k^{0}\right) Q(k) b^{ \pm d}(k)=0, \\
& \pm i \theta\left(k^{0}\right)\left[k^{\lambda} a_{\lambda}^{ \pm d}(k)+(V \cdot k) V^{\lambda} a_{\lambda}^{ \pm d}(k)\right]=\theta\left(k^{0}\right) \xi b^{ \pm d}(k) .
\end{aligned}
$$

Inserting (80) into (77) gives the commutation relations

$$
\left[a_{\lambda}^{+d}(k), a_{\mu}^{+^{c}}(q)\right]=0,\left[a_{\lambda}^{-d}(k), a_{\mu}^{-c}(q)\right]=0
$$

and

$$
\begin{aligned}
{\left[a_{\sigma}^{-d}(p), a_{\tau}^{+c}(q)\right]=} & -\mu\left(g_{\sigma \tau}-\frac{\beta}{1+\beta} V_{\sigma} V_{\tau}\right) \delta^{d c} \delta(Q(p)) \delta(p-q) \\
& -(\mu-\xi) \delta^{d c} p_{\sigma} p_{\tau} \delta^{\prime}(Q(p)) \delta(p-q)
\end{aligned}
$$


For the operators $b^{ \pm d}(p)$ one finds

$$
\left[b^{+d}(p), b^{+c}(q)\right]=0,\left[b^{-d}(p), b^{-c}(q)\right]=0
$$

and

$$
\left[a_{\lambda}^{-d}(p), b^{+c}(q)\right]=-\delta^{d c} p_{\lambda} \delta(Q(p)) \delta(p-q) .
$$

It is convenient for each four vector $p$ to introduce a local frame consisting of the orthonormal vectors $V,\left(V^{2}=1\right), N(p)$ and $e^{(r)}(p),(r=1,2)$, satisfying

$$
N(p)^{2}=\left(e^{(r)}(p)\right)^{2}=-1,
$$

where

$$
N(p)_{\mu} \equiv \frac{\tilde{p}_{\mu}-(V \cdot \tilde{p}) V_{\mu}}{\sqrt{(V \cdot \tilde{p})^{2}-\tilde{p}^{2}}}
$$

and $\tilde{p}_{\mu} \equiv p_{\mu}+\beta V_{\mu}(V \cdot p)$. We decompose the operators $a_{\lambda}^{ \pm d}(p)$ with respect to the base vectors $V, N(p), e^{(r)}(p)$,

$$
a_{\lambda}^{ \pm d}(p)=\sum_{r=1}^{2} \alpha_{r}^{ \pm d}(p) e_{\lambda}^{(r)}(p)+\alpha_{3}^{ \pm d} N_{\lambda}+\alpha_{0}^{ \pm d} V_{\lambda} .
$$

The commutation relations for the new operators $\alpha_{\lambda}^{ \pm d}(p)$ read

$$
\begin{aligned}
& {\left[\alpha_{0}^{-d}(p), \alpha_{0}^{+c}(q)\right]=-\frac{\mu \delta^{d c}}{1+\beta} \delta(Q(p)) \delta(p-q)-(\mu-\xi) \delta^{d c}(V \cdot p)^{2} \delta^{\prime}(Q(p)) \delta(p-q),} \\
& {\left[\alpha_{3}^{-d}(p), \alpha_{3}^{+^{c}}(q)\right]=\mu \delta^{d c} \delta(Q(p)) \delta(p-q)-(\mu-\xi) \delta^{d c}\left[(V \cdot p)^{2}-p^{2}\right] \delta^{\prime}(Q(p)) \delta(p-q),} \\
& {\left[\alpha_{k}^{-d}(p), \alpha_{l}^{+^{c}}(q)\right]=\mu \delta^{d c} \delta(Q(p)) \delta(p-q)} \\
& {\left[\alpha_{0}^{-d}(p), \alpha_{3}^{+c}(q)\right]=-(\mu-\xi) \delta^{d c} \sqrt{(V \cdot p)^{2}-p^{2}}(V \cdot p) \delta^{\prime}(Q(p)) \delta(p-q),} \\
& {\left[\alpha_{0}^{-d}(p), \alpha_{k}^{+c}(q)\right]=0} \\
& {\left[\alpha_{3}^{-d}(p), \alpha_{k}^{+c}(q)\right]=0} \\
& {\left[\alpha_{k}^{-d}(p), b^{+c}(q)\right]=0} \\
& {\left[\alpha_{3}^{-d}(p), b^{+c}(q)\right]=-i \delta^{d c} \sqrt{(V \cdot p)^{2}-p^{2}} \delta(Q(p)) \delta(p-q)} \\
& {\left[\alpha_{0}^{-d}(p), b^{+c}(q)\right]=-i \delta^{d c}(V \cdot p) \delta(Q(p)) \delta(p-q) .}
\end{aligned}
$$

The vacuum vector $|0\rangle$ of the Fock space $\mathcal{H}_{F}$ is defined by

$$
\alpha_{\lambda}^{-d}(p)|0\rangle=0 \text {. }
$$

It follows from the commutation relations (82)-(90) that the operators $\alpha_{0}^{+d}(p)$ and $\alpha_{3}^{+d}(p)$ do not generate states with positive definite norm squared due to the terms with $\delta^{\prime}(Q(p))$. The physical subspace of $\mathcal{H}_{F}$ is defined by

$$
\mathcal{H}_{\text {phys }}=\left\{|\Psi\rangle \in \mathcal{H}_{F} ; b^{-d}(p)|\Psi\rangle=0\right\} .
$$

According to equation (81) the condition $b^{-d}(p)|\Psi\rangle=0$ is equivalent to

$$
\tilde{p} a_{\lambda}^{-d}(p)|\Psi\rangle=0
$$


implying

$$
L^{-d}(p)|\Psi\rangle=0
$$

where

$$
L^{ \pm d}(p)=\sqrt{(V \cdot p)^{2}-p^{2}} \alpha_{0}^{ \pm d}(p)-(V \cdot p) \alpha_{3}^{ \pm d}(p)
$$

for any $|\Psi\rangle \in \mathcal{H}_{\text {phys }}$. The states with non-negative norm squared are generated by $\alpha_{1,2}^{+d}(p)$ and $L^{+d}(p)$ which are the operators commuting with $b^{-c}(q)$. However, the subspace $\mathcal{H}_{0}$ of $\mathcal{H}_{\text {phys }}$ generated by $L^{+d}(p)$ consists of zero norm vectors. Therefore, the space of vectors with positive norm squared is defined by the quotient

$$
\mathcal{H}^{\prime}=\mathcal{H}_{\text {phys }} / \mathcal{H}_{0} .
$$

The energy-momentum tensor $T_{\lambda \mu}$ derived from the Lagrangian $\mathcal{L}=\mathcal{L}_{0}+\mathcal{L}_{g f}$ is

$$
T_{\lambda \mu}=-\mathcal{H}_{\lambda \tau}^{a} \partial_{\mu} A^{a, \tau}+\left(b^{-2} \cdot A^{a}\right)_{\lambda} \partial_{\mu} B^{a}-g_{\lambda \mu} \mathcal{L},
$$

$\left(b^{-2}\right)_{\lambda}^{\sigma}=\left(\delta_{\lambda}^{\sigma}+\beta V_{\lambda} V^{\sigma}\right)$, and may be written as

$$
T_{\lambda \mu}=-\mathcal{H}_{\lambda \tau}^{a} F_{\mu}^{a, \tau}+\frac{1}{4} g_{\lambda \mu}\left(F_{\alpha \beta}^{a} H^{a, \beta \alpha}\right)
$$

up to a total divergence $\sim \partial^{\tau}\left(\mathcal{H}_{\lambda \tau}^{a} A_{\mu}^{a}\right)$. For the matrix elements of the corresponding four-momentum operator one finds

$$
\left\langle\Phi\left|P_{\lambda}\right| \Phi^{\prime}\right\rangle=\int d^{3} k k_{\lambda}\left\langle\Phi\left|\sum_{r=1}^{2} \alpha_{r}^{+^{a}}(k) \alpha_{r}^{-a}(k)\right| \Phi^{\prime}\right\rangle,
$$

$|\Phi\rangle,\left|\Phi^{\prime}\right\rangle \in \mathcal{H}^{\prime}$

In the gauge $\xi=\mu$ the free propagator $\left\langle 0\left|T\left(A_{\mu}^{b}(x) A_{\nu}^{d}(y)\right)\right| 0\right\rangle$ reads

$$
(1 / i) \mathcal{D}_{\mu \nu}^{b d}(x-y) \equiv\left\langle 0\left|T\left(A_{\mu}^{b}(x) A_{\nu}^{d}(y)\right)\right| 0\right\rangle=-i \mu\left(g_{\mu \nu}-\frac{\beta}{1+\beta} V_{\mu} V_{\nu}\right) \delta^{b d} F_{c}(x-y),
$$

where

$$
F_{c}(x-y)=\int \frac{d^{4} k}{(2 \pi)^{4}} \frac{e^{-i k(x-y)}}{Q(k)+i \epsilon} .
$$

As a short example of applications of the theory we shall consider two phenomenas that are possible to exist in reactions at high energies.

For the specific case $G=S U(3)$ and quarks as fermions, the $\beta /(1+\beta)$ term in the gluon propagator (91) modifies, e.g. the cross-sections for quark-quark scattering. For instance, the expression for elastic scattering of two unpolarized quarks of mass $m$ and initial (final) momenta $p, k,\left(p^{\prime}, k^{\prime}\right)$ in the Born approximation is

$$
\begin{aligned}
\frac{d \sigma}{d \Omega}= & \frac{2 \alpha_{s}^{2}}{s}\left[\frac{F_{0}}{Q^{2}\left(p-p^{\prime}\right)}+\frac{H_{0}}{Q^{2}\left(p-k^{\prime}\right)}-\frac{2 G_{0}}{3 Q\left(p-p^{\prime}\right) Q\left(p-k^{\prime}\right)}\right]- \\
& -\frac{\beta}{1+\beta} \frac{2 \alpha_{s}^{2}}{s}\left[\frac{F_{1}}{Q^{2}\left(p-p^{\prime}\right)}+\frac{H_{1}}{Q^{2}\left(p-k^{\prime}\right)}-\frac{2 G_{1}}{3 Q\left(p-p^{\prime}\right) Q\left(p-k^{\prime}\right)}\right]+ \\
& +\frac{\beta^{2}}{(1+\beta)^{2}} \frac{2 \alpha_{s}^{2}}{s}\left[\frac{F_{2}}{Q^{2}\left(p-p^{\prime}\right)}+\frac{H_{2}}{Q^{2}\left(p-k^{\prime}\right)}-\frac{2 G_{2}}{3 Q\left(p-p^{\prime}\right) Q\left(p-k^{\prime}\right)}\right],
\end{aligned}
$$


where we set the permeability $\mu=1$ and

$$
\begin{aligned}
& F_{0}=16 s^{2}+16 s t+8 t^{2}-64 m^{2} s+64 m^{4}, \\
& H_{0}=8 s^{2}+8 t^{2}-64 m^{2} s-64 m^{2} t+192 m^{4}, \\
& G_{0}=-8 s^{2}+64 s m^{2}-96 m^{4} \\
& F_{1}=32\left\{(p V)\left[(k V)\left(p^{\prime} k^{\prime}\right)+\left(k^{\prime} V\right)\left(p^{\prime} k\right)\right]+\left(p^{\prime} V\right)\left[\left(k^{\prime} V\right)(p k)+(k V)\left(p k^{\prime}\right)\right]-\right. \\
& \left.-2(p V)\left(p^{\prime} V\right)\left(k k^{\prime}\right)-2(k V)\left(k^{\prime} V\right)\left(p p^{\prime}\right)\right\}+32\left(p p^{\prime}\right)\left(k k^{\prime}\right)+ \\
& +32 m^{2}\left[2(p V)\left(p^{\prime} V\right)+2(k V)\left(k^{\prime} V\right)-\left(p p^{\prime}\right)-\left(k k^{\prime}\right)\right]+32 m^{4}, \\
& H_{1}=32\left\{(p V)\left[(k V)\left(p^{\prime} k^{\prime}\right)+\left(p^{\prime} V\right)\left(k k^{\prime}\right)\right]+\left(k^{\prime} V\right)\left[\left(p^{\prime} V\right)(p k)+(k V)\left(p p^{\prime}\right)\right]-\right. \\
& \left.-2(p V)\left(k^{\prime} V\right)\left(k p^{\prime}\right)-2(k V)\left(p^{\prime} V\right)\left(p k^{\prime}\right)\right\}+32\left(p k^{\prime}\right)\left(k p^{\prime}\right)+ \\
& +32 m^{2}\left[2(p V)\left(k^{\prime} V\right)+2(k V)\left(p^{\prime} V\right)-\left(p k^{\prime}\right)-\left(k p^{\prime}\right)\right]+32 m^{4}, \\
& G_{1}=16 m^{2}\left[(p V)(k V)+\left(p^{\prime} V\right)\left(k^{\prime} V\right)\right]+4\left[(p+k)\left(p^{\prime}+k^{\prime}\right)-2 m^{2}\right]- \\
& -8(p k)\left(p^{\prime} k^{\prime}\right) \\
& F_{2}=64(p V)\left(p^{\prime} V\right)(k V)\left(k^{\prime} V\right)-32(p V)\left(p^{\prime} V\right)\left(k k^{\prime}\right)-32(k V)\left(k^{\prime} V\right)\left(p p^{\prime}\right)+ \\
& +32 m^{2}\left[(p V)\left(p^{\prime} V\right)+(k V)\left(k^{\prime} V\right)\right]-16 m^{2}\left[\left(p p^{\prime}\right)+\left(k k^{\prime}\right)\right]+ \\
& +16\left(p p^{\prime}\right)\left(k k^{\prime}\right)+16 m^{4} \\
& H_{2}=64(p V)\left(p^{\prime} V\right)(k V)\left(k^{\prime} V\right)-32(p V)\left(k^{\prime} V\right)\left(k p^{\prime}\right)-32(k V)\left(p^{\prime} V\right)\left(p k^{\prime}\right)+ \\
& +32 m^{2}\left[(p V)\left(k^{\prime} V\right)+(k V)\left(p^{\prime} V\right)\right]-16 m^{2}\left[\left(p k^{\prime}\right)+\left(k p^{\prime}\right)\right]+16\left(p k^{\prime}\right)\left(k p^{\prime}\right)+ \\
& +16 m^{4} \\
& G_{2}=32(p V)\left(p^{\prime} V\right)(k V)\left(k^{\prime} V\right)-8(p V)\left[\left(p^{\prime} V\right)\left(k k^{\prime}\right)+\left(k^{\prime} V\right)\left(p^{\prime} k\right)\right]- \\
& -8(k V)\left[\left(k^{\prime} V\right)\left(p p^{\prime}\right)+\left(p^{\prime} V\right)\left(p k^{\prime}\right)\right]+4\left(p p^{\prime}\right)\left(k k^{\prime}\right)-4(p k)\left(p^{\prime} k^{\prime}\right)+ \\
& +4\left(p k^{\prime}\right)\left(p^{\prime} k\right) \text {. }
\end{aligned}
$$

The first line in the expression for the cross-section is (for $n=1$ ) the known result from QCD in the Born approximation (see e.g. [36]).

Another change comes from the term $\beta(k \cdot V)^{2}$ in each denominator $Q(k)=$ $k^{2}+\beta(k \cdot V)^{2}$. For very large $\beta=n^{2}-1$, this term may lead to an effective decreasing of the coupling strength. 
As a second example we consider the in-medium Yang-Mills equations to describe the gluon field emitted by a quark moving in a medium. If $j_{\mu}^{a}(x)$ is a non-zero quark current in the r.h.s. in (62), the corresponding gauge potential $A_{\mu}(x)$ satisfies the equation (in the gauge $\xi=\mu$ )

$$
\left(\square+\beta(V \cdot \partial)^{2}\right) A_{\mu}^{a}(x)=j_{\mu}^{a}(x)
$$

for a medium with "refraction" coefficient $n=\sqrt{\beta^{2}+1}$. In the $V$-rest frame the equation becomes

$$
\left(n^{2} \frac{\partial^{2}}{\partial x_{0}^{2}}-\triangle\right) A_{\mu}^{a}(x)=j_{\mu}^{a}(x)
$$

and for a "rapid" quark, a Cherenkov-type gluon radiation will appear. Thus the description presented here provides a natural framework for the Dremin's approach aimed at explaining events (such as ring-like structures) which occur in hadron matter at high energies.

\section{Summary}

In this paper the in-medium Yang-Mills equations are derived for a gauge group $G$ in the case of a linear response to an external gauge field. As in classical electromagnetism, there appears naturally a conductivity tensor accompanied, however, by additional nonAbelian conductivity tensor and pseudotensor originating from the non-commutative character of the gauge group $G$. Correspondingly, the medium is characterized by "electric" and "magnetic" polarizations as well as by a new type of non-Abelian "electric" and "magnetic" polarizations. When the latter are assumed to be linearly related with the fields $E^{a}$ and $B^{a}$, the in-medium equations refer to the generalized "electric" and "magnetic" inductions and for fields $E^{\prime a}$ and $B^{\prime a}$ modified by the appearing susceptibility tensors. If, however, these non-Abelian polarizations depend non-linearly on $E^{a}$ and $B^{a}$, the restriction to a linear response reduces the modified fields to $E^{a}$ and $B^{a}$, and the in-medium Yang-Mills equations become similar to the macroscopic Maxwell equations. In an approximation up to second order in the gauge coupling constant $g$, the in-medium equations resemble the macroscopic Maxwell equations for $\operatorname{dim}(G)$ copies of the electromagnetic field. In this case the medium characteristics - the conductivity tensor $\sigma$, the electric permittivity tensor $\epsilon$ and the magnetic permeability tensor $\mu$ are expressed in terms of retarded Green's functions in a way analogous to the case of the in-medium electrodynamics. The canonical quantization is performed for constant and diagonal $\epsilon$ and $\mu$ in order to simplify the Euler-Lagrange equations. A set of $\operatorname{dim}(G)$ scalar auxiliary fields $B^{a}$ is introduced to avoid working with constrained systems. The commutation relations among the gauge potentials $A_{\mu}^{a}$ and the field $B^{a}$ are derived in two ways for a family of Fermi-like gauges. The first one is straightforward but lengthy and employs an integral representation of the solutions of equation (75), while the second one is direct and based on a simple transformation of the both coordinates and 
fields. For the gauge choice $\xi=\mu$ we write down the "gluon" propagator $\mathcal{D}_{\mu \nu}^{b d}$ and evaluate the cross-section for elastic scattering of two identical unpolarized quarks in the Born approximation. A feature of the propagator is the appearance of the term $\beta(k . V)^{2}$ which may lead to an effective decrease of the coupling strength for sufficiently large "refraction" coefficient. It is pointed out that the in-medium Yang-Mills equations allow to match Dremin's description of the above mentioned ring-like structures.

\section{References}

[1] G. K. Savvidy, Phys. Lett. B 71 (1977) 133.

[2] R. Fukuda and Y. Kazama, Phys. Rev. Lett. 45 (1980) 1142.

[3] S. L. Adler, Phys. Rev. D 23 (1981) 2905.

[4] H. Nielsen and P. Olsen, Nucl. Phys. B 144 (1978) 376.

[5] M. Baker and F. Zachariasen, Phys. Lett. B 108 (1982) 206.

[6] M. Baker and F. Zachariasen, Nucl. Phys. B 226 (1983) 455.

[7] D. Sivers, Phys. Rev. D 27 (1983) 947.

[8] J. Ralston and D. Sivers, Phys. Rev. D 28 (1983) 953.

[9] U. Heinz, Am. Phys. 161 (1985) 48.

[10] U. Heinz, Am. Phys. 168 (1986) 148.

[11] Chen Jisheng, Zheng Xiaoping and Li Jiarong, The nonlinear permittivity including non-Abelian self-interaction of plasmons in QGP, Chin. Phys. Lett. 17 (2000) 714.

[12] Ji-sheng Chen, Jia-rong Li and Peng-fei Zhuang, Non-Abelian medium effects in QGP, Int. J. Mod. Phys. A 17 (2002) 1435.

[13] D. F. Litim and C. Manuel, Phys. Rev. Lett. 82 (1999) 4981.

[14] D. F. Litim and C. Manuel, Nucl. Phys. B 562 (1999) 237.

[15] D. F. Litim and C. Manuel, Phys. Rep. 364 (2002) 451.

[16] D. H. Rischke, D. T. Son and M. A. Stephanov, Phys. Rev. Lett. 87 (2001) 062001.

[17] D. Litim and C. Manuel, Phys. Rev. D 64 (2001) 094013.

[18] J. Ruppert, G. Nayak, D. Dietrich, H. Stöcker and W. Greiner, Phys. Lett. B 520 (2001) 233.

[19] D. F. Litim and C. Manuel, Phys. Rev. Lett. 87 (2001) 052002.

[20] R. Friedberg and T. D. Lee, Phys. Rev. D 15 (1977) 1694.

[21] R. Friedberg and T. D. Lee, Phys. Rev. D 16 (1977) 1696.

[22] U. Kalmbach, T. Vetter, T. S. Biró and U. Mosel, Nucl. Phys. A 563 (1993) 584.

[23] T. Vetter, T. S. Biró and U. Mosel, Phys. Lett. A 581 (1995) 598.

[24] S. Loh, T. S. Biró, U. Mosel and M. H. Thoma, Phys. Lett. B 387 (1996) 685.

[25] C. T. Traxler, U. Mosel and T. S. Biró, Phys. Rev. C 59 (1999) 1620.

[26] G. Martens, C. Traxler, U. Mosel and T. S. Biró, Hadronization in the chromodielectric model, hep-ph/0002037.

[27] D. Bazeia, F. A. Brito, W. Freire, R. F. Ribeiro, Confining potential in a color dielectric model with paralel domain walls, hep-th/0210289.

[28] I.M. Dremin, JETP Lett. 30 (1979) 140.

[29] I.M. Dremin, Yad. Fiz.33 (1981) 1357.

[30] I.M. Dremin, Symmetries and Wavelets, hep-ph/0108229.

[31] A. I. Akhiezer and S. V. Peletminsky, Methods of Statistical Physics, Pergamon, London, 1981.

[32] I. Brevik and B. Lautrup, Quantum Electrodynamics in Material Media. Mat. Fys. Medd. Dan. Vid. Selsk. 38, (1970) NORDITA.

[33] N. Nakanishi, Progr. Theor. Physics, 35 (1966) 1111.

[34] N. Nakanishi, Suppl. Progr. Theor. Physics, 51 (1972) 1.

[35] D. Zwanziger, Phys. Rev. D 17 (1978) 457. 
[36] H. Bohr, J. Phys. G 9 (1983) 373. 